\title{
Heterogeneous Nucleation and Microstructure Formation: From a Model Systems to Applied Metal Physics
}

\author{
HEIKE EMMERICH ${ }^{1,2}$ \\ 1.-Chair for Materials and Process Simulation, Bayreuth University, Bayreuth, Germany. \\ 2.—e-mail: sekretariat-mps@uni-bayreuth.de
}

Most physical and chemical properties of metallic alloys are determined to a large degree via their microstructure morphology. One example is the crystal structure of a ferritic and an austinitic steel, respectively, in which the desired mechanical, electrical, and magnetic properties are strongly tied to a likewise desired specific microstructure of the steel. As many of today's industrial relevant metallic alloy systems are processed from the molten state, a most quantitative understanding of solidification as an essential primary processing step is a premise for a most quantitative tailoring of such desired microstructures. Nonetheless, heterogeneous nucleation and successive microstructure formation as initial solidification steps are still far from being comprehensively understood. However, the comparison of available advanced models and recent experimental results brings about controversial conclusions. ${ }^{1-3}$

To progress these scientific discussions, from 2007 to 2013, the German Research Foundation (DFG) funded the scientific priority program 1296 related to the heterogeneous nucleation and initial microstructure formation, ${ }^{4}$ aiming at a fundamental understanding of the basic mechanisms underlying heterogeneous nucleation as well as the subsequent development of the nucleus into a specific heterogeneous microstructure. To that end, a system- and method-spanning scientific approach was developed, in which the simplest types of model systems for heterogeneous crystalline orders, pure metals, binary metal alloys, and colloids were investigated in a comparative manner. Moreover, they were assessed with complementary experimental as well as simulation techniques from the atomic to the microscale to obtain a comprehensive view across all relevant time and length scales, as depicted in Fig. 1.

Heike Emmerich is the guest editor for the Phase Transformations Committee of the TMS Materials Processing \& Manufacturing Division, and coordinator of the topic Heterogeneous Nucleation and Initial Microstructural Formation in this issue.
Some questions explored in detail in the context of the priority program were as follows: With respect to heterogeneous nucleation, what does a critical nucleation grain look like? Does the classic concept of a contact angle make sense for heterogeneous nucleation? Can claims made about the dominant contributions to the nucleation barrier for heterogeneous nucleation, such as they can be obtained from molecular simulations, be reconciled with data gained via the phase-field method? With respect to transition from nucleus to microstructure, how does a microstructure develop from a nucleus in the interplay between crystallization and segregation depending on the precise reference point in the phase diagram? How stable are those scenarios with respect to changes of that reference point? With respect to microstructure development, what kind of consequence results from the new understanding of nucleation for the initial development of the microstructure, e.g., in terms of new kinetic scaling laws? What kind of kinetic rules does the initial growth of the solidifying microstructure follow?

An overview of the most important results of that priority program has recently been presented in Ref. 5.

In this section of $J O M$ we go a step further and present some of the more applied recent results acquired in the priority program, which build the bridge from fundamental investigations in model systems toward industrially more relevant multicomponent and multiphase metallic systems. The more applied specific questions related to heterogeneous nucleation and initial microstructure formation in such systems addressed here range from the identification of the quantitative influence of inoculation process parameters on the amount of achievable grain refinement, over a comprehensive assessment of the kinetics in four-phase peritectic reactions, pointing out differences in different types of peritectica, to a quantitative assessment of nucleation energies in solid-solid systems based on newly established simulation methodologies. In to- 


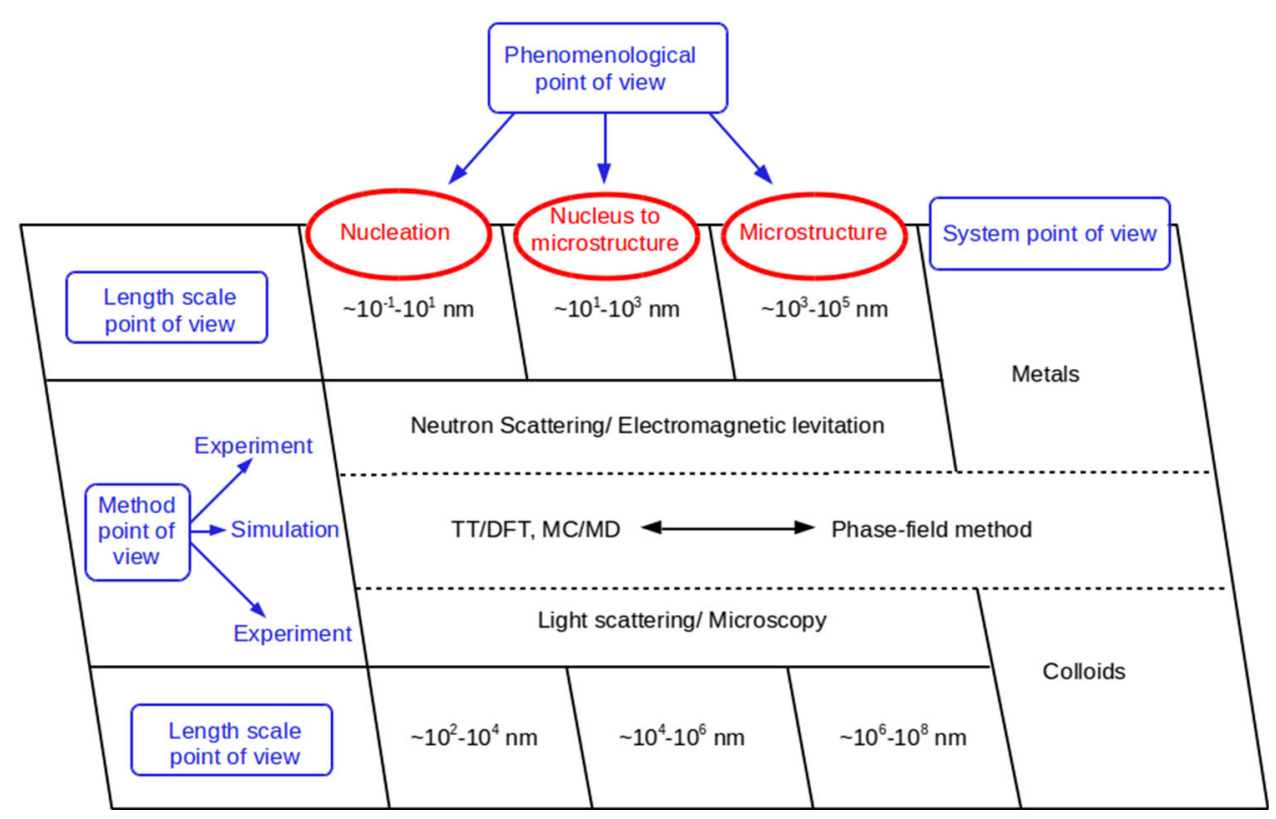

Fig. 1. Schematics of the interaction of different theoretical and experimental techniques to access heterogeneous nucleation kinetics and dynamics most comprehensively over several time and length scales. The figure illustrates the key idea of the priority program, namely to exploit a smearing of scales as given by the two complementary material systems for a most comprehensive assessment of the diverse kinetic, energetic, and morphological features involved in heterogeneous nucleation. Reprinted from Ref. 5.

tal, this JOM focus section includes four articles with the following emphasis:

The first article by Gosslar and Günther ${ }^{6}$ is focused on grain size model calculations of titanium diboride inoculated titanium aluminide based alloys. The model uses the free growth criterion of grain initiation upon heterogeneous nucleation, which implies that grains initiate once their interfacial curvature reaches a maximum. The presented calculations identify quantitatively the influence of inoculation process parameters on the amount of achievable grain refinement. A benchmark test against experimental results allows the discussion of the grain size reduction efficiency of titanium diboride inoculants.

In a second article by Kundin et al. ${ }^{7}$ the peculiarities of phase transitions and structure formation in a ternary $\mathrm{Al}-\mathrm{Cu}-\mathrm{Ni}$ alloy with four-phase peritectic reaction are assessed. To that end, the structure formation in a peritectic Al-4.5at.\% Cu11 at.\% Ni ternary alloy with four-phase peritectic reaction was investigated using a quantitative phase-field model of eutectic growth in tight comparison with experiments. Based on that, quantitative new insight into the kinetics of four-phase peritectic reactions, elucidating differences in different types of peritectica, is achieved and presented in comparison with classic Scheil predictions.

In a fully experimental approach toward heterogeneous nucleation and initial microstructure formation in more component metallic alloys, Wilde et al. ${ }^{8}$ demonstrated one way to circumvent ambiguities and analyze nucleation kinetics. Application of proper statistical analysis yields nucleation rates that are independent of a specific nucleation model. First studies that were conducted in accordance with this approach on pure model materials demonstrate the validity of the approach. The results are comparable with those obtained by classic nucleation theory applied to experimental data indicating that one might need to rethink the common assumption that heterogeneous nucleation is almost always responsible for the initiation of solidification. The current results also show that often used models for the solid-liquid interface free energy might lead to overestimated values.

Finally Chen et al. ${ }^{9}$ briefly review recent advances in modeling and predicting nucleation during solid phase transformations based on a diffuse-interface or nonclassic description of critical nucleus profiles. The focus is on predicting the critical nucleus morphology and nucleation free energy barrier under the influence of anisotropic interfacial energy and elastic interactions. Ways to incorporate nucleation events in phase-field modeling of solid-to-solid phase transformations and microstructure evolution are presented as well.

With the presentation of these four articles, our aim is to point out possible transfer directions that continue the bridge from fundamental nucleation and microstructure related investigations in model systems with the above focus toward more applied multicomponent and multiphase metallic systems.

\section{REFERENCES}

1. R. Willnecker, D.M. Herlach, and B. Feuerbacher, Mater. Sci. Eng. A 98, 85 (1988). 
2. L. Gránásy, J. Non-Cryst. Solids 162, 301 (1993).

3. K.I. Dragnevski, R.F. Cochrane, and A. Mullis, Mater. Sci. Eng. A 375, 479 (2004).

4. Homepage of the SPP 1296. Heterogeneous Nucleation and Microstructure Formation. http://www.spp1296.uni-bayr euth.de/en/index.html. Accessed 24 Apr 2014.

5. H. Emmerich, P. Virnau, G. Wilde, and R. Spatschek, Eur. Phys. J. Special-Top. 223, 337 (2014).
6. D. Gosslar and R. Günther, JOM 66 (2014). doi:10.1007/ s11837-014-1031-y.

7. J. Kundin, H. Emmerich, P. Wang, and R. Schmid-Fetzer, JOM 66 (2014). doi:10.1007/s11837-014-1052-6.

8. J. Bokeloh and G. Wilde, JOM (2014). doi:10.1007/s11837014-1027-7.

9. T.W. Heo and L.Q. Chen, JOM (2014). doi:10.1007/s11837014-1033-9. 